\title{
Transfer of abductor hallucis tendon combined with scarf osteotomy versus single scarf osteotomy in moderate to severe hallux valgus deformity: a comparative retrospective cohort study
}

\author{
Yuan Xiong ${ }^{\dagger}$, Bo Shen ${ }^{\dagger}$, Cheng Hao, Kai Xiao, Junwen Wang and Zhenhua Fang ${ }^{*}$
}

\begin{abstract}
Background: Scarf osteotomy (SO) was broadly applied in moderate to severe hallux valgus (MSHV), and the results were satisfactory. However, due to the complicated pathologic changes in hallux valgus, the ideal surgical treatment is still controversial. Transfer of the abductor hallucis tendon combined with Scarf osteotomy (TAHTCSO) was an innovative alternative technique. This retrospective cohort study aimed to define if TAHTCSO mode resulted in improved outcomes as compared with the single SO in MSHV.

Methods: Of 73 patients $(92 \mathrm{ft}$ ) with MSHV, $36(45 \mathrm{ft})$ were treated through TAHTCSO and 37 ones $(47 \mathrm{ft})$ through SO. The patients were assessed clinically and radiographically with a 24-month follow-up. They were assessed preoperatively and post-operatively with intermetatarsal angle (IMA), hallux valgus angle (HVA), distal metatarsal articular angle (DMAA), first metatarsophalangeal joint range of motion (1\#MTP ROM), as well as American Orthopaedic Foot and Ankle Society (AOFAS) forefoot scores and postoperative complications of surgery.

Results: Both cohorts had the same baseline feathures. All patients were followed up from 24 to 40 months, with a mean of 28.3 months. Patients in the TAHTCSO cohort had significantly decreased HVA at 6 months $(p<0.0001), 12$ months $(p<0.0001)$, and 24 months $(p<0.0001)$ after surgery. 1\#MTP had been increased slightly with non-statistic sense $(p>0.05)$. IMA, DMAA and AOFAS also had not significantly difference at all followed time after surgery as compared with the SO cohort. The healing of osteotomies was observed within 8 weeks in the two cohorts. Two cases of hallus varus had been found in $\mathrm{SO}$ cohort and there were no cases of delayed healing and bone nonunion in both cohorts.
\end{abstract}

Conclusion: In this retrospective cohort study, TAHTCSO had sufficient maintenance of the correction and improved functional performance thereby was a good alternative for MSHV, though it did not display a better result for MSHV compared to SO.

Keywords: Hallux valgus, Musculus abductor hallucis tendon, Scarf osteotomy

\footnotetext{
* Correspondence: foot_ankle_surgeon@163.com

†Yuan Xiong and Bo Shen contributed equally to this work

Department of Orthopaedics, Wuhan Fourth Hospital, Puai Hospital, Tongji

Medical College, Huazhong University of Science and Technology, Wuhan

43033, China
}

(c) The Author(s). 2019 Open Access This article is distributed under the terms of the Creative Commons Attribution 4.0 International License (http://creativecommons.org/licenses/by/40/) which permits unrestricted use, distribution, and reproduction in any medium, provided you give appropriate credit to the original author(s) and the source, provide a link to the Creative Commons license, and indicate if changes were made. The Creative Commons Public Domain Dedication waiver (http://creativecommons.org/publicdomain/zero/1.0/) applies to the data made available in this article, unless otherwise stated. 


\section{Introduction}

Moderate to severe hallux valgus deformity is defined as intermetatarsal angle (IMA) of more than 13 degrees [1]. It often occurs in the aged population with assessed occurrence of $36 \%$ in people over 65 years [2]. Some cases combined with other foot deformities, such as lesser toe, hindfoot or midfoot deformities may exacerbate the pathology. For MSHV correction, a variety of treatment options including foot orthoses, soft tissue release, and osteotomies are described [35]. However, the unique and most appropriate MSHV treatment remain elusive. The diaphyseal osteotomies such as Scarf osteotomy have gained popularity in recent years [6]. It provides excellent inherent biomechanical stability and allows early weight-bearing. In patients with MSHV, Scarf osteotomy seems to be a preferred surgery [7].

Abductor hallucis muscle plays an important role in maintaining first metatarsophalangeal joint stability and preventing abnormal transverse plane motion [8]. Transfer of the abductor hallucis tendon in hallux valgus surgery is a valuable addition in correcting the pronation deformity of the great toe [9]. TAHTCSO is an innovative alternative fixation approach (Fig. 1). This retrospective cohort study aimed to investigate if TAHTCSO results in improved outcomes as compared with single SO method.

\section{Materials and methods}

\section{Patient eligibility}

This is a retrospective study comparing the effects of TAHTCSO and SO in MSHV patients. From July 2013 to July 2015, 73 patients $(92 \mathrm{ft}$ ) with MSHV were treated surgically and assessed retrospectively. Ethical approval and informed written consent were obtained from every single patient. Eligible patients were included in this research once they met the following criteria: (1) Diagnosed with MSHV (IMA more than 13 degrees), (2) were over 18 years of old and in full possession of their mental faculties, (3) had severe pain, (4) patients treated with TAHTCSO were included in TAHTCSO group or treated with SO were included in SO group, and were follow-up until 24 months, (5) first metatarsal osteotomy by a single surgeon, and (6) symptomatic hallux valgus associated with increased DMAA $\left(\geq 15^{\circ}\right)$.

\section{Surgical treatment and the rehabilitation protocol}

Surgically, all patients were treated by a single, senior surgeon for correcting deformity, relieving severe pain, and rebuilding inherent biomechanical stability. A medial incision is made, subcutaneous tissue was dissected bluntly in order to protect the medial dorsal neuro-vascular bundle. In TAHTCSO Cohort, the abductor hallucis tendon was be exposed, and 1/2 2/3 tendinous insertion of the abductor hallucis muscle was released from its ends and be transferred toward dorsal of the medial capsule, which can not only strengthen the medial tension but also achieve dynamic reconstruction of the medial soft tissue. The tendon was stitched with the capsule directly (Fig. 2). In both cohorts, patients were subjected to the same postoperative rehabilitation protocol, which allowed them to walk as much as they could on the heel side of the foot by a post-operative shoe after 3 days of surgery. Active and assisted motion of the metatarsophalangeal joint was permitted when suigical wounds were healed. Forefoot bandage and post-operative shoe were used for 4 and $6 \sim 8$ weeks, respectively.

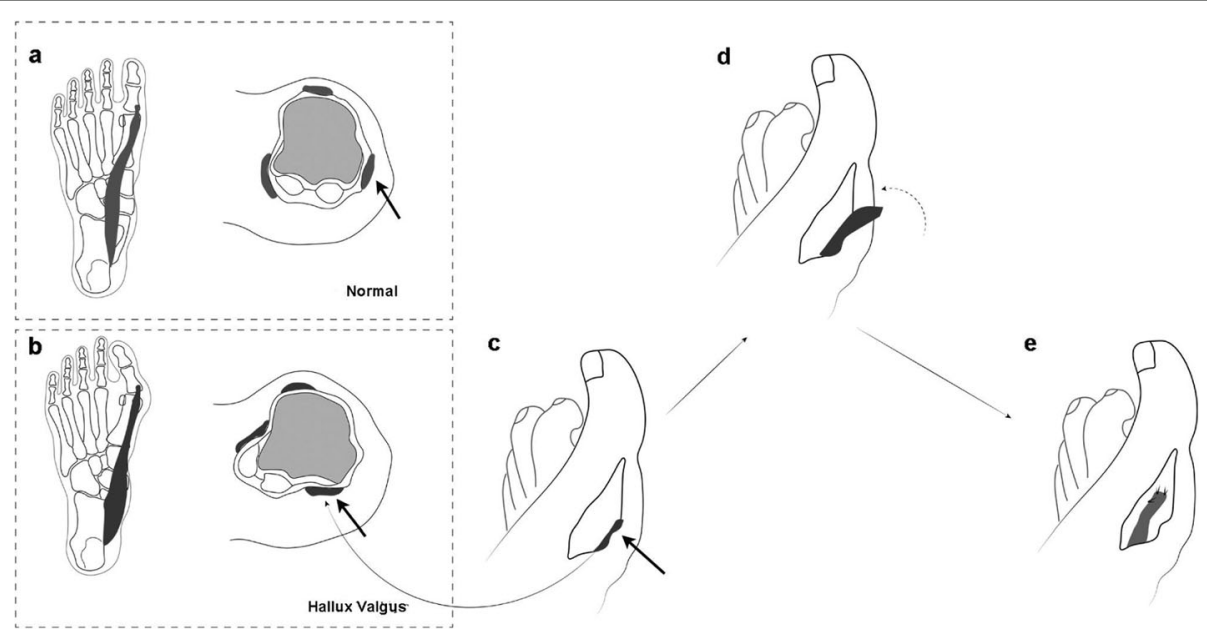

Fig. 1 Transposition of abductor hallucis tendon. a the normal position of abductor hallucis. b abductor hallucis shift more plantar. c-e transposition of abductor hallucis tendon. Black arrow represents abductor hallucis 

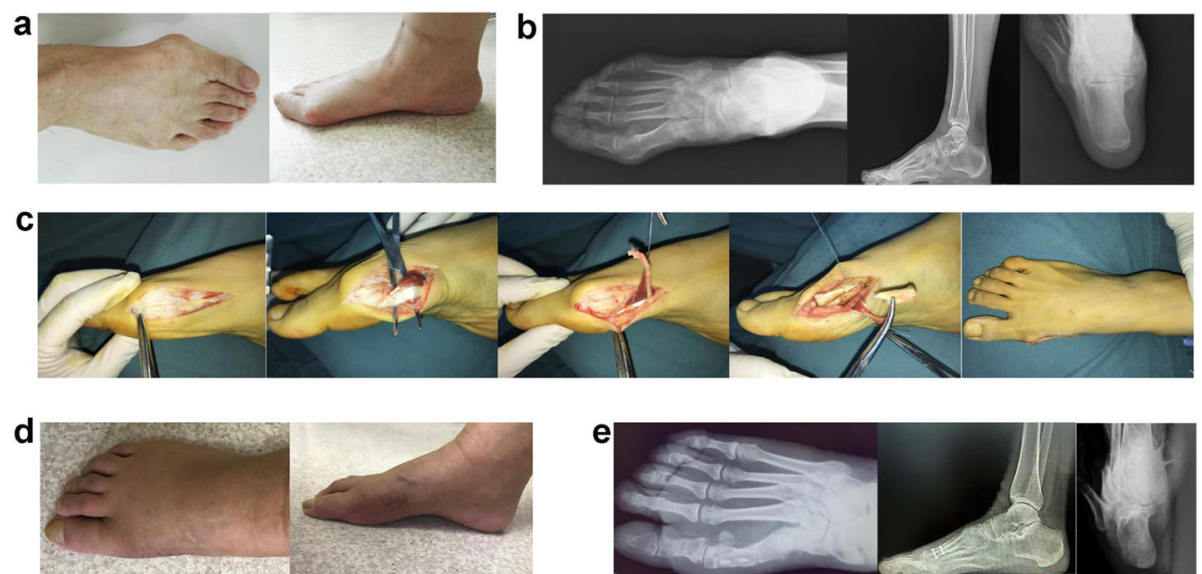

Fig. 2 A 69-year-old woman had right-sided hallux valgus deformity. a to b Digital photos and X-ray showing the hallux valgus deformity. c The process of surgical technique. $\mathbf{d}$ Digital photos and X-ray after 24-month follow up

\section{Patient assessment}

The baseline features comprising age, sex, BMI, and VAS pain score were collected. IMA, HVA, DMAA, and 1 \#MTP ROM were collected prospectively at predetermined intervals of 6,12 and 24 months post-operatively. Subjective clinical results were measured through the AOFAS score which is one of the broadly applied clinician-reporting tools for forefoot and ankle illness. AOFAS is a clinician-based score that measures effects on four various anatomic regions of the foot: the anklehindfoot, midfoot, metatarsophalangeal (MTP)-interphalangeal (IP) for the hallux, and MTP-IP for the lesser toes. In our investigation, complications were also gathered until the 24 months' follow-up.

\section{Statistical analysis}

All statistical analyses were carried out via IBM SPSS Statistical software (version 22, IBM, Somers, NY, USA). The data was provided as the mean \pm standard deviation (SD) for continuous variables and as numbers for categorical measures. The paired sample $t$-test was applied to compare the measurements before and after the surgery. $P<0.05$ was regarded as an important difference.

\section{Results}

In this research, 36 patients $(45 \mathrm{ft})$ treated with TAHT $\mathrm{CSO}$ and 37 ones $(47 \mathrm{ft})$ treated with $\mathrm{SO}$ were taken part. The two cohorts had the same baseline features comprising mean age $(65.9+10.4$ and $66.8+10.5$ years, $p=0.5132$ ), gender distribution (male: female, $\mathrm{n}, 24: 21$ and 20:27, $p=0.0817)$, BMI $(\mathrm{kg} / \mathrm{m} 2,27.4+3.9$ and $29.0+6.4, p=0.0526)$, and mean VAS pain score $(87.2$ and 92.9, $p=0.4133$ ) (Table 1). Patients in the TAHT CSO cohort had significantly decreased HVA at 6 months $(p<0.0001), 12$ months $(p<0.0001)$ and 24 months $(p<0.0001)$ after the surgery as compared with the SO cohort. Patients in the TAHTCSO cohort, had 1\#MTP increased slightly with non-statistic sense $(p>$ 0.05). IMA, DMAA and AOFAS also had not a significantly difference at all followed time after surgery as compared with the SO cohort. The healing of osteotomies was observed within 8 weeks in the two cohorts. Two cases of hallus varus and three cases of troughing were found in SO cohor; there were no cases of delayed healing and bone non-union in both cohorts (Table 2).

\section{Discussion}

During recent years, the most commonly used alternative to MSHV is the SO that provides adequate contact between metaphyseal bone surfaces, prevents shortening of the first metatarsal and allows early mobilization [10, 11]. Adequate correction of the hallux valgus is obtained by greater rotation and translation of the plantar-distal fragment $[12,14]$. Although SO is relatively easy for fixation, and our study did not find insufferable complications, it is technically demanding that can lead to worsening of symptoms if non-union or avascular necrosis of the first metatarsal head occurs [9, 11, 13]. In addition, the plantar fragment excessive rotation may result in an increase in the DMAA that can contribute to a lateral hallux deviation [8]. Although SO is identified by many surgeons for hallux valgus, it is far from a

Table 1 The baseline characters of patients

\begin{tabular}{llll}
\hline & $\operatorname{TAHTCSO}(n=45)$ & $\mathrm{SO}(n=47)$ & $P$ value \\
\hline Age, y, mean \pm SD & $65.9 \pm 10.4$ & $66.8 \pm 10.5$ & .5132 \\
Sex, male:female, $\mathrm{n}$ & $24: 21$ & $20: 27$ & .0817 \\
BMI, kg/m2, mean \pm SD & $27.4 \pm 3.9$ & $29.0 \pm 6.4$ & .0526 \\
VAS pain score (0-100), mean & 87.2 & 92.9 & .4133
\end{tabular}

SO Scarf osteotomy, TAHTCSO Transfer of the abductor hallucis tendon combined with Scarf osteotomy, BMI Body mass index, VAS Visual analog scale 
Table 2 Clinical and radiographic assessment results of patients with MSHV

\begin{tabular}{|c|c|c|c|c|}
\hline & $\operatorname{TAHTCSO}(n=45)$ & $\mathrm{SO}(n=47)$ & $P$ & Test \\
\hline IMA before surgery & $16.47 \pm 2.30$ & $15.98 \pm 1.89$ & 0.3472 & Unpaired $T$ test \\
\hline IMA 6 months & $7.03 \pm 0.70$ & $7.27 \pm 1.35$ & 0.1405 & Unpaired T test \\
\hline IMA 12 months & $7.31 \pm 1.72$ & $8.03 \pm 3.67$ & 0.2301 & Unpaired $T$ test \\
\hline IMA 24 months & $8.62 \pm 2.49$ & $9.02 \pm 2.43$ & 0.2505 & Unpaired T test \\
\hline HVA before surgery & $34.02 \pm 3.49$ & $33.02 \pm 2.43$ & 0.9072 & Unpaired $T$ test \\
\hline HVA 6 months* & $11.96 \pm 1.33$ & $15.72 \pm 3.99$ & $<0.0001$ & Unpaired T test \\
\hline HVA 12 months* & $13.03 \pm 1.70$ & $17.11 \pm 3.69$ & $<0.0001$ & Unpaired $T$ test \\
\hline HVA 24 months* & $13.71 \pm 2.62$ & $19.38 \pm 2.53$ & $<0.0001$ & Unpaired T test \\
\hline DMAA before surgery & $9.71 \pm 2.62$ & $9.38 \pm 2.53$ & 0.4589 & Unpaired $T$ test \\
\hline DMAA 6 months & $6.09 \pm 0.89$ & $6.32 \pm 0.77$ & 0.0542 & Unpaired $T$ test \\
\hline DMAA 12 months & $6.23 \pm 1.02$ & $6.25 \pm 1.15$ & 0.1537 & Unpaired $T$ test \\
\hline DMAA 24 months & $7.05 \pm 1.13$ & $7.12 \pm 0.89$ & 0.2769 & Unpaired $T$ test \\
\hline 1\#MTP ROM (dorsiflexion) before surgery & $68.25 \pm 2.90$ & $68.73 \pm 3.10$ & 0.3681 & Unpaired $T$ test \\
\hline 1\#MTP ROM (dorsiflexion) 6 months & $69.23 \pm 3.02$ & $69.16 \pm 1.89$ & 0.1364 & Unpaired $T$ test \\
\hline 1\#MTP ROM (dorsiflexion) 12 months & $71.18 \pm 2.81$ & $70.09 \pm 2.23$ & 0.2246 & Unpaired $T$ test \\
\hline 1\#MTP ROM (dorsiflexion) 24 months & $73.78 \pm 2.72$ & $75.08 \pm 1.87$ & 0.3013 & Unpaired T test \\
\hline 1\#MTP ROM (plantarflexion) before surgery & $33.05 \pm 3.13$ & $32.12 \pm 2.89$ & 0.2589 & Unpaired T test \\
\hline 1\#MTP ROM (plantarflexion) 6 months & $35.72 \pm 2.97$ & $36.73 \pm 1.78$ & 0.1854 & Unpaired $T$ test \\
\hline 1\#MTP ROM (plantarflexion) 12 months & $36.63 \pm 1.98$ & $37.03 \pm 1.89$ & 0.2583 & Unpaired T test \\
\hline 1\#MTP ROM (plantarflexion) 24 months & $37.73 \pm 2.08$ & $39.71 \pm 1.06$ & 0.2641 & Unpaired T test \\
\hline AOFAS before surgery & $44.01 \pm 0.56$ & $43.24 \pm 0.57$ & 0.4709 & Unpaired $T$ test \\
\hline AOFAS 6 months & $76.67 \pm 0.19$ & $76.61 \pm 0.15$ & 0.3821 & Unpaired $T$ test \\
\hline AOFAS 12 months & $80.17 \pm 2.15$ & $81.64 \pm 0.19$ & 0.3531 & Unpaired $T$ test \\
\hline AOFAS 24 months & $80.69 \pm 0.19$ & $79.67 \pm 0.39$ & 0.1432 & Unpaired T test \\
\hline Complication hallus varus & 0 & 2 & 0.0856 & Fisher's exact test \\
\hline Complication delayed healing & 0 & 0 & - & - \\
\hline Complication nonunion & 0 & 0 & - & - \\
\hline Complication troughing & 0 & 3 & 0.0512 & Fisher's exact test \\
\hline Additional Akin osteotomy (n) & 9 & 12 & 0.0631 & Fisher's exact test \\
\hline
\end{tabular}

panacea [14]. Traditional SO simply releases the tendinous insertion of the adductor hallucis muscle and tightens the medial capsule. However, for MSHV with abnormal large HVA degree, strengthening the medial capsule is not enough to accomplish complete correction.

The major result of the current study is the efficacy of TAHTCSO for a novel approach. Abductor hallucis is located medial to the first metatarsal, which arises from the medial process of the calcaneal tuberosity and inserts on the medial surface of the base of the first proximal phalanx. It is the single muscle that directly may keep the hallux from abducting (displacing laterally) deformity [3]. In patients with hallux valgus, the abductor tendon shifts plantarward that contributes to the joint deformity $[10,12]$. Despite the important role of the abductor hallucis muscle in the pathology of the deformity, few studies have examined the transfer of abductor hallucis muscle for hallux valgus correction [15]. The aim of the abductor hallucis transfer is to stabilize the medial soft tissue components of the first metatarsophalangeal joint [15]. In this study, part of the abductor hallucis tendon is released from its ends and transfered it toward dorsal of the medial capsule, which can not only strengthen the medial tension but also provide a dynamic reconstruction of the medial soft tissue. Transfer of the abductor hallucis tendon is important to restore the physiological situation and the first metatarsophalangeal joint 
function. This technique was recommended by our good clinical results in a whole follow up.

Akin osteotomy is often used to accompany scarf osteotomy. Kristen reported that Akin osteotomy is added after completing the scarf osteotomy and reconstructing soft tissue when the hallux is still greater than $10^{\circ}$ of valgus position [7]. In other researches, Akin osteotomy has been reported to be used in 70 to $100 \%$ of cases after Scarf osteotomy $[4,6]$. Unlike previous studies, only $9 \mathrm{ft}$ were added an additional Akin osteotomy in TAHTCSO cohort. This may contribute to the fact that Scarf osteotomy combined with transfer of abductor hallucis tendon provides good correction for MSHV with no need to an Akin osteotomy; it suggested that the transfer of abductor hallucis muscle has strengthened the medial tension and developed a dynamic reconstruction of the medial soft tissue, and then can prevent HVA lost after operation. Troughing is another complication of Scarf osteotomy that takes place once the cortices wedge into the cancellous bone of the metatarsal shaft, which in turn result in stiffness of the first metatarsophalangeal joint [1]. Some studies found that it can be prevented by shifting the rotation of the osteotomy or transposition of the removed cortical bone between osteotomy sites [11, 13]. In TAHTCSO group, there was no troughing case, which may be contributed to the transposition of the removed cortical bone between osteotomy sites. Basically, the tendon transfer did not affect the troughing phenomenon. The troughing cases in TAHTCOSO may be contributed to some factors as below: 1 . osteoporosis in patients; 2 . the tendon transposition may intensify the HV correction ability and decrease the bone transposition to assure the cortical bone contraction. We also agree that TAHT is one of the most factors for causing iatrogenic varus. In present research, the reversed results may contribute to two main aspects. Firstly, although all the operations were performed by a same surgeon in this retrospective study, there were still some subtle differences in the intensity and degree of osteotomy among individual patients, which might lead to some differences that were not statistically significant. The other hand is that, the samples included in this study is limited, which is also the limitation of present retrospective study. In the follow-up study, we will further expand the sample size of the study, and throw high light on observing the differences of hallux valgus and other complications among groups.

Quantification analysis of the range of abductor hallucis tendon transfer has not been reported in previous studies. In the present study, 1/2 2/3 part of tendon of abductor halluces was released from its ending points and shifted towards dorsal of the first metatarsal capsule, which provided satisfying clinical result in the 24-month follow-up. However, for the long-term efficacy and potential complications (such as the residue abductor hallucis tendon rupture, and hallux varus), further studies are still needed to verify this surgical technique.

The strength of this study is that all surgeries were carried out by a single senior author. The clinical and radiographic data were assessed by three independent investigators respectively. The limitation of this study is that it was a retrospective study, with a few patients included. In addition, a 24-month follow-up can only be regarded as a short-term follow-up, which failed to provide information regarding the long-term consequences of the procedure. Furthermore, the patients with osteoporosis, osteoarthritis of the first metatarsophalangeal joint and so forth were excluded.

Above all, TAHTCSO is an effective technique for MSHV deformity. Although some cases with residual valgus need additional Akin osteotomies, all patients were satisfied with the function of their feet. The most important aspects that recommend the application of this method are its low number of complications, early weight-bearing, as well as good clinical and radiographic assessment results.

\section{Conclusions}

In this retrospective cohort study, TAHTCSO had sufficient maintenance of the correction and improved functional performance, thereby a good alternative for MSHV, though it did not display a better results for MSHV as compared with SO.

\section{Abbreviations \\ AOFAS: American Orthopaedic Foot and Ankle Society; DMAA: Distal metatarsal articular angle; HVA: Hallux Valgus Angle; IMA: Intermetatarsal Angle; MSHV: Moderate to severe hallux valgus; MTP \\ ROM: Metatarsophalangeal joint range of motion; SO: Scarf osteotomy; TAHT CSO: Transfer of the abductor hallucis tendon combined with Scarf osteotomy}

\section{Acknowledgements}

This work was accomplished with the help of the library of Huazhong University of Science and Technology.

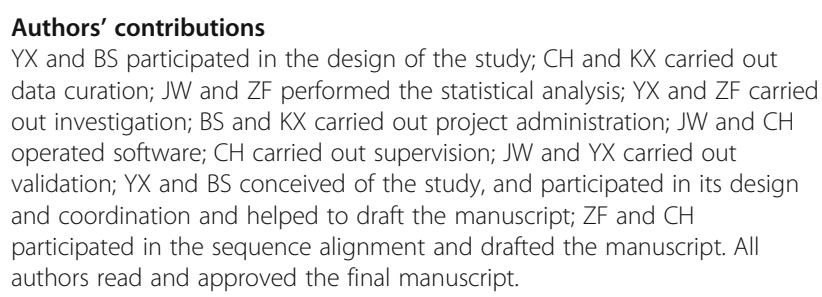

Funding

No external funding was received for the initiation or completion of this study.

Availability of data and materials

Please contact author Zhenhua Fang for data requests. 


\section{Ethics approval and consent to participate}

The study was approved by the clinical research ethics committee of Wuhan Puai Hospital. Reference No. 2013-049-02. And informed written consent was obtained from every single patient before receiving surgery.

\section{Consent for publication}

Not applicable.

\section{Competing interests}

The authors declare that they have no competing interests.

Received: 4 September 2018 Accepted: 1 October 2019

Published online: 20 October 2019

\section{References}

1. Adam SP, Choung SC, Gu Y, O'Malley MJ. Outcomes after scarf osteotomy for treatment of adult hallux valgus deformity. Clin Orthop Relat Res. 2011; 469(3):854-9.

2. Agrawal Y, Bajaj SK, Flowers MJ. Scarf-Akin osteotomy for hallux valgus in juvenile and adolescent patients. J Pediatr Orthop B. 2015;24(6):535-40.

3. Arinci Incel N, Genc H, Erdem HR, Yorgancioglu ZR. Muscle imbalance in hallux valgus: an electromyographic study. Am J Phys Med Rehabil. 2003; 82(5):345-9.

4. Crevoisier X, Mouhsine E, Ortolano V, Udin B, Dutoit M. The scarf osteotomy for the treatment of hallux valgus deformity: a review of 84 cases. Foot Ankle Int. 2001:22(12):970-6.

5. Curtin M, Murphy E, Bryan C, et al. Scarf osteotomy without internal fixation for correction of hallux valgus: A clinical and radiographic review of 148 cases.[J] Foot Ankle Surg. 2018;24:252-58. PMID: 29409250.

6. Jones S, Al Hussainy HA, Ali F, Betts RP, Flowers MJ. Scarf osteotomy for hallux valgus. A prospective clinical and pedobarographic study. J Bone Joint Surg Br. 2004;86(6):830-6.

7. Kristen KH, Berger C, Stelzig S, Thalhammer E, Posch M, Engel A. The SCARF osteotomy for the correction of hallux valgus deformities. Foot Ankle Int. 2002;23(3):221-9.

8. Larholt J, Kilmartin TE. Rotational scarf and akin osteotomy for correction of hallux valgus associated with metatarsus adductus. Foot Ankle Int. 2010; 31(3):220-8.

9. Leemrijse T, Hoang B, Maldague P, Docquier PL, Devos BB. A new surgical procedure for iatrogenic hallux varus: reverse transfer of the abductor hallucis tendon: a report of 7 cases. Acta Orthop Belg. 2008;74(2):227-34

10. Kamila M, Przemysław L, Agnieszka WK. The study of surface electromyography used for the assessment of abductor hallucis muscle activity in patients with hallux valgus. [J] Physiother Theory Pract. 2018;34: 846-51. PMID:29373047.

11. Murawski CD, Egan CJ, Kennedy JG. A rotational scarf osteotomy decreases troughing when treating hallux valgus. Clin Orthop Relat Res. 2011;469(3): $847-53$

12. Uğur S, Budak A, Altuğ T, et al. The results of Scarf osteotomy combined with distal soft tissue procedure are mostly satisfactory in surgical management of moderate to severe hallux valgus.[J] Foot Ankle Surg. 2018; 24:448-52. PMID:29409200.

13. Schoen NS, Zygmunt K, Gudas C. Z-bunionectomy: retrospective long-term study. J Foot Ankle Surg. 1996;35(4):312-7.

14. Tsikopoulos K, Papaioannou P, Kitridis D, Mavridis D, Georgiannos D. Proximal versus distal metatarsal osteotomies for moderate to severe hallux valgus deformity: a systematic review and meta-analysis of clinical and radiological outcomes. Int Orthop. 2018;42(8):1853-63.

15. Waizy H, Stukenborg-Colsman C, Abbara-Czardybon M, Emmerich J, Windhagen $\mathrm{H}$, Frank D. A special soft tissue procedure for treatment of hallux valgus. Oper Orthop Traumatol. 2011;23(1):46-51.

\section{Publisher's Note}

Springer Nature remains neutral with regard to jurisdictional claims in published maps and institutional affiliations.

\section{Ready to submit your research? Choose BMC and benefit from:}

- fast, convenient online submission

- thorough peer review by experienced researchers in your field

- rapid publication on acceptance

- support for research data, including large and complex data types

- gold Open Access which fosters wider collaboration and increased citations

- maximum visibility for your research: over $100 \mathrm{M}$ website views per year

At BMC, research is always in progress.

Learn more biomedcentral.com/submissions 